\title{
UBE2D3 is a positive prognostic factor and is negatively correlated with hTERT expression in esophageal cancer
}

\author{
GE GE GUAN $^{1 *}$, WEN BO WANG ${ }^{1,2^{*}}$, BING XIN LEI $^{1}$, QIAO LI WANG ${ }^{1}$, LIN WU $^{1}$, \\ ZHEN MING FU ${ }^{1,2}$, FU XIANG ZHOU ${ }^{1,2}$ and YUN FENG ZHOU ${ }^{1,2}$ \\ ${ }^{1}$ Hubei Key Laboratory of Tumor Biological Behaviors, Hubei Cancer Clinical Study Center; \\ ${ }^{2}$ Department of Radiation and Medical Oncology, Zhongnan Hospital, Wuhan University, Wuhan, Hubei 430071, P.R. China
}

Received September 3, 2014; Accepted January 13, 2015

DOI: $10.3892 / \mathrm{ol} .2015 .2926$

\begin{abstract}
Human telomerase reverse transcriptase (hTERT) is a critical factor in unlimited cell proliferation and immortalization, with numerous studies demonstrating that high expression of hTERT is a poor prognostic factor in various types of cancer. Ubiquitin-conjugating enzyme E2D 3 (UBE2D3) is a member of the E2 family, and participates in the ubiquitin proteasome pathway to regulate basic cellular activities, such as cell cycle control, the DNA damage response, apoptosis, and tumorigenesis. Our previous study initially determined that downregulation of UBE2D3 expression increases hTERT expression and cell proliferation, however, the association between the expression of these two proteins and their functions in cancer tissues remains unknown. Therefore, the protein expression levels of hTERT and UBE2D3 were evaluated in 150 esophageal cancer and 30 adjacent healthy tissue samples by performing immunohistochemical analysis. Concurrently, the clinicopathological data of the enrolled patients were obtained to allow correlation analysis. It was identified that the expression of hTERT in the esophageal cancer tissues was significantly higher compared with that of the adjacent tissues $(\mathrm{P}=0.015)$, however, the expression of UBE2D3 was significantly lower in esophageal cancer tissues than the adjacent tissues $(\mathrm{P}=0.001)$. Additionally, the study demonstrated that hTERT was significantly upregulated in poorly-differentiated, advanced tumor-node-metastasis (TNM) stage cancer tissues $(\mathrm{P}<0.05$ for all), however, UBE2D3 expression was downregulated in poorly-differentiated, lymph node invaded
\end{abstract}

Correspondence to: Professor Yun Feng Zhou, Department of Radiation and Medical Oncology, Zhongnan Hospital of Wuhan University, 169 Donghu Road, Wuchang, Wuhan, Hubei 430071, P.R. China

E-mail: yfzhouwhu@163.com

*Contributed equally

Key words: ubiquitin-conjugating enzyme E2D 3, human telomerase reverse transcriptase, esophageal cancer, prognosis cancer tissues and recurrent cases. It was also identified that traditional factors, including tumor location, T stage, lymph node status, TNM stage, and molecular factors of hTERT and UBE2D3, were significantly associated with overall survival time $(\mathrm{P}<0.05$ for all). Furthermore, UBE2D3, lymph node status and tumor location were independent prognostic factors for esophageal cancer in multivariate analysis. Most notably, hTERT and UBE2D3 expression were negatively correlated with each other. In conclusion, the findings of the present study indicated that hTERT and UBE2D3 proteins appear to be involved in the development of esophageal cancer, that UBE2D3 may a positive prognostic factor for esophageal cancer, and that UBE2D3 and hTERT expression levels are inversely correlated.

\section{Introduction}

Esophageal cancer is the eighth most common type of cancer and ranks as the sixth leading cause of cancer-related mortality worldwide $(1,2)$. In particular, China contains areas of the highest incidences of esophageal cancer in the world, with esophageal cancer classified as the sixth most frequently diagnosed type of cancer (3) and the fourth most common cause of cancer mortality in China (4). In the previous two decades, comprehensive therapeutic regimes aimed at improving survival have been widely applied, however, the overall five-year relative survival rate is just $16.9 \%$ (5) due to the aggressive nature of this type of malignancy. Currently, the traditional tumor-node-metastasis (TNM) classification systems of the International Union Against Cancer (UICC) and American Joint Committee on Cancer are the most important tools used for predicting the prognosis of esophageal cancer patients and the only method used on a routine basis (6). However, esophageal cancer is highly heterogeneous, and tumors with the same TNM stage demonstrate differences in clinical course and treatment response. Thus, the identification of factors to predict malignant potential and prognosis is of great importance.

Telomeres are unique structures composed of double-stranded tandem repeats of TTAGGG at the end of a chromosome (7). Telomerase, a ribonucleoprotein enzyme, consists of a catalytic protein unit, human telomerase reverse transcriptase (hTERT), and human telomerase RNA. Together, 
the two units synthesize telomeric sequences that allow tumor cells to escape from cellular senescence when reactivated or are upregulated by tumor cells to allow indefinite proliferation (8). The expression of hTERT closely correlates with telomerase activity and serves as an indicator of telomerase activation (9,10). Although previous studies have demonstrated that high hTERT expression is a predictive and prognostic biomarker of a poor outcome in a range of malignancies, including Ewing's sarcoma, and colorectal, gastric and breast carcinoma (11-14), studies regarding the role of hTERT expression in esophageal cancer are sparse and thus warrant further investigation.

The ubiquitin proteasome pathway (UPP) and the lysosome degradation system are two distinctive proteolytic mechanisms required for intracellular protein degradation (15). The UPP participates in protein quality control via the degradation of misfolded proteins (16) in order to regulate basic cellular activities, such as cell cycle control, the DNA damage response, apoptosis and tumorigenesis (17). The functioning of the UPP is achieved by the coordinated action of a cascade of three enzyme species: Ubiquitin activating enzymes (E1), ubiquitin-conjugating enzymes (E2) and ubiquitin ligases (E3) $(16,18)$. Ubiquitin-conjugating enzymeE2D3(UBE2D3), also known as UBCH5C, is a member of the E2 family. In our previous study, a yeast two-hybrid screen was performed to identify that a mutual association exists between hTERT and the hTERT-interacting protein UBE2D3; downregulation of UBE2D3 resulted in the accumulation of hTERT, indicating that UBE2D3 potentially has a role in the hTERT signaling pathway (19). Thus, the aim of the present study was to elucidate the clinical significance of hTERT and UBE2D3 expression in esophageal cancer.

\section{Patients and methods}

Patients and follow-up. The esophageal cancer tissue specimens were obtained from patients who had undergone curative surgery (esophagectomy with lymph node dissection) at the Zhongnan Hospital of Wuhan University (Wuhan, Hubei, China) between January 2006 and December 2012. No patients had previously received palliative resection, pre-operative chemotherapy or radiotherapy, and no patients exhibited synchronous or metachronous cancer in other organs or succumbed in the month following surgery. All post-operative specimens were diagnosed with esophageal cancer by two pathologists and the TNM stage was determined according to the seventh edition of the UICC TNM system (20).

According to the aforementioned criteria, 150 patients were included in the present study. Follow-up data were available for all patients on a one to three month basis, the most recent follow-up date was June 10, 2014, and the median follow-up time was 37 months (range, 14-90 months). In addition, a total of $91(60.7 \%)$ patients succumbed during the follow-up period. Any recurrence in the anastomotic mediastinum, esophagus or regional lymph nodes was defined as local-regional recurrence, and recurrence identified via blood flow was defined as distant metastasis, such as liver, lung and bone metastasis. Overall survival (OS) time was defined as the period between the date of the surgical procedure and the date of mortality. Furthermore, the present study was conducted in accordance with the Declaration of Helsinki of the World Medical Association and the protocols were approved by the Ethics Committee of Zhongnan Hospital of Wuhan University, with consent obtained from all patients.

Immunohistochemical analysis and evaluation. Immunohistochemical staining was performed using the streptavidin-biotin method to detect hTERT and UBE2D3 protein expression levels. Firstly, the $4 \mu \mathrm{m}$-wide sections of esophageal cancer and adjacent tissues were incubated at room temperature for $60 \mathrm{~min}$, followed by exposure to dimethylbenzene for $10 \mathrm{~min}$. The sections were then deparaffinized in 100, 95 and $75 \%$ ethyl alcohol for $5 \mathrm{~min}$, respectively. Next, the tissue samples were microwaved in citrate buffer (0.1 mol/1, pH 6.0) for $10 \mathrm{~min}$ at $1,000 \mathrm{~W}$. Endogenous peroxidase was blocked by incubating the sections in $3 \%$ hydrogen peroxide for $5 \mathrm{~min}$ at room temperature. Subsequent to washing with distilled water and incubating with goat serum (Fuzhou Maixin Biotechnology Development Co., Ltd., Fuzhou, China) for $1 \mathrm{~h}$, the sections were incubated with primary monoclonal hTERT (cat no., ab32020; dilution, 1:100; Abcam, Cambridge, MA, USA) or polyclonal UBE2D3 (cat no., 11677-1-AP; dilution, 1:50; Proteintech Group, Inc., Chicago, IL, USA) antibodies at room temperature for $2 \mathrm{~h}$. Biotinylated secondary goat anti-rabbit antibodies and peroxidase-conjugated streptavidin obtained from the DAKO Universal LSAB ${ }^{\mathrm{TM}}$ kit (Dako, Glostrup, Denmark) were applied for $20 \mathrm{~min}$ each. Finally, the sections were incubated in 3'3'-diaminobenzidine for $5 \mathrm{~min}$, followed by hematoxylin counterstaining. Two negative controls were performed by replacing the primary antibody with non-immune serum and omitting the application of the secondary antibody.

The intensity of the immunostaining was evaluated using light microscopy. Two independent investigators, who were blinded to the clinicopathological data, evaluated the levels of protein expression in each section, and the staining intensity was scored as follows: No staining, 0; weak staining, 1; moderate staining, 2; and strong staining, 3 . Additionally, the extent of staining was scored according to the percentage of positively-stained cells in the entire carcinoma-involved area, as follows: Absent, 0; sporadic, 1-10\%; local, 11-25\%; occasional, 26-50\%; majority, 51-75\%; and large majority, 76-100\%. For hTERT expression, an intensity score of $\geq 2$ and $\geq 11 \%$ of cells with positive hTERT staining was considered to indicate high expression, and an intensity score of $<2$ or $<11 \%$ of cells with hTERT positive staining was considered to indicate low expression (21). By contrast, UBE2D3 protein expression levels were classified as high when UBE2D3 staining was present in $>50 \%$ of cells (22). Expression in the cytoplasm and nucleus was regarded as an indication of positive expression for the two proteins $(22,23)$. Using this method, the expression of hTERT and UBE2D3 was detected in 150 esophageal cancer and 30 adjacent tissue samples.

Statistical analysis. Statistical analyses were performed using SPSS software (version 17.0; SPSS, Inc., Chicago, IL, USA). The Pearson $\chi^{2}$ test or Fisher's exact test were used to 
compare qualitative variables. Kaplan-Meier analysis was performed for univariate analysis and significance among patient subgroups was calculated using the log-rank test. Furthermore, the Cox proportional hazard model was used to conduct multivariate analysis and Spearman correlation coefficients were applied for analyzing the association between the expression of the two proteins. Two-sided $\mathrm{P}<0.05$ was considered to indicate a statistically significant difference.

\section{Results}

Major clinicopathological features and immunohistochemical findings. Included in the present study were 145 samples of squamous cell cancer and five other pathological types. The age range of the patients was 39-85 years (mean \pm stan-

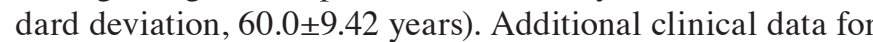
the current patients are presented in Table I.

Immunohistochemical analysis of all esophageal cancer and healthy adjacent tissues was performed. According to the aforementioned classification system, 11 healthy adjacent tissue samples with high hTERT expression and 21 with high UBE2D3 expression were identified, compared with 91 esophageal cancer tissue samples with high hTERT expression and 55 with high UBE2D3 expression. Thus, the expression levels of these two proteins demonstrated a significant difference between the cancerous and adjacent tissues $(\mathrm{P}=0.015$ and $\mathrm{P}=0.001$, respectively). Fig. 1A-D shows representative examples of hTERT expression in esophageal cancer samples and Fig. 1E-H shows representative examples of UBE2D3 expression. According to the aforementioned staining intensity and extent scores, Fig. 1A, B, E and F demonstrated high expression levels, however, Fig. 1C, D, G and H demonstrated low UBE2D3 expression levels.

Association between expression levels of hTERT and UBE2D3, and various clinicopathological features. As summarized in Table II, hTERT expression was significantly higher in larger-sized, poorly-differentiated, and advanced $\mathrm{T}, \mathrm{N}$ and $\mathrm{TNM}$ stage tumors $(\mathrm{P}=0.039, \mathrm{P}=0.032$, $\mathrm{P}=0.030, \mathrm{P}=0.018$ and $\mathrm{P}=0.012$, respectively). Furthermore, UBE2D3 expression levels were significantly lower in poorly-differentiated, advanced $\mathrm{N}$ stage and recurrent cases of esophageal cancer $(\mathrm{P}=0.007, \mathrm{P}=0.016$ and $\mathrm{P}=0.008$, respectively).

Survival analysis. In the 150 cases, the median OS time was 20.0 months (range, 5-84 months), and the one-, three- and five-year survival rates were $72.3,40.7$ and $25.0 \%$, respectively. As indicated in Fig. 2, a number of traditional factors were associated with the OS time of the esophageal cancer patients, including tumor location, fibrous membrane invasion, lymph node status and TNM stage $(\mathrm{P}<0.05$ for all; Fig. 2A-D). In addition, low expression levels of hTERT and high expression levels of UBE2D3 were associated with improved $\mathrm{OS}$ time $(\mathrm{P}=0.012$ and $\mathrm{P}=0.002$, respectively; Fig.2E and F).

Multivariate analysis. Univariate analysis (Table III) indicated that tumor location, T, N and TNM stage, and hTERT and UBE2D3 expression may predict esophageal cancer
Table I. Patient demographics and clinicopathological characteristics.

\begin{tabular}{|c|c|}
\hline Characteristic & $\mathrm{n}(\%)$ \\
\hline \multicolumn{2}{|l|}{ Age, years } \\
\hline$<60$ & $70(46.7)$ \\
\hline$\geq 60$ & $80(53.3)$ \\
\hline \multicolumn{2}{|l|}{ Gender } \\
\hline Male & $124(82.7)$ \\
\hline Female & $26(17.3)$ \\
\hline \multicolumn{2}{|l|}{ Location } \\
\hline Upper thoracic & $21(14.0)$ \\
\hline Middle/lower thoracic & $129(86.0)$ \\
\hline \multicolumn{2}{|l|}{ Tumor size, $\mathrm{cm}$} \\
\hline$<5$ & $81(54.0)$ \\
\hline$\geq 5$ & $69(46.0)$ \\
\hline \multicolumn{2}{|l|}{ Histological grade ${ }^{a}$} \\
\hline $\mathrm{G} 1 / 2$ & $119(79.3)$ \\
\hline G3/G4 & $31(20.7)$ \\
\hline \multicolumn{2}{|l|}{ T stage } \\
\hline $\mathrm{T} 1-2$ & $65(43.3)$ \\
\hline T3-4 & $85(56.7)$ \\
\hline \multicolumn{2}{|l|}{ N stage } \\
\hline No & $76(50.7)$ \\
\hline N1-3 & $74(49.3)$ \\
\hline \multicolumn{2}{|l|}{ TNM stage } \\
\hline Early (I-II) & $88(58.7)$ \\
\hline Advanced (III-IV) & $62(41.3)$ \\
\hline \multicolumn{2}{|l|}{ Adjuvant radiotherapy } \\
\hline Yes & $40(26.7)$ \\
\hline No & $110(73.3)$ \\
\hline \multicolumn{2}{|l|}{ Adjuvant chemotherapy } \\
\hline Yes & $70(46.7)$ \\
\hline No & $80(53.3)$ \\
\hline \multicolumn{2}{|l|}{ Recurrence } \\
\hline No & $66(44.0)$ \\
\hline Yes & $84(56.0)$ \\
\hline \multicolumn{2}{|l|}{ Recurrence location } \\
\hline Local-regional & $50(59.5)$ \\
\hline Distant & $34(40.5)$ \\
\hline
\end{tabular}

${ }^{\mathrm{a}} \mathrm{G} 1 / 2$ were defined as well- or moderately-differentiated and G3/4 were defined as poorly-differentiated or undifferentiated. TNM, tumor-node-metastasis.

prognosis, therefore, these factors were integrated into multivariate analysis using Cox proportional hazards analysis. It was subsequently identified that tumor location, lymph node status and UBE2D3 expression level were independent prognostic factors in esophageal cancer (Table IV).

Correlation between hTERT and UBE2D3 expression levels. The Spearman correlation coefficient for hTERT and 

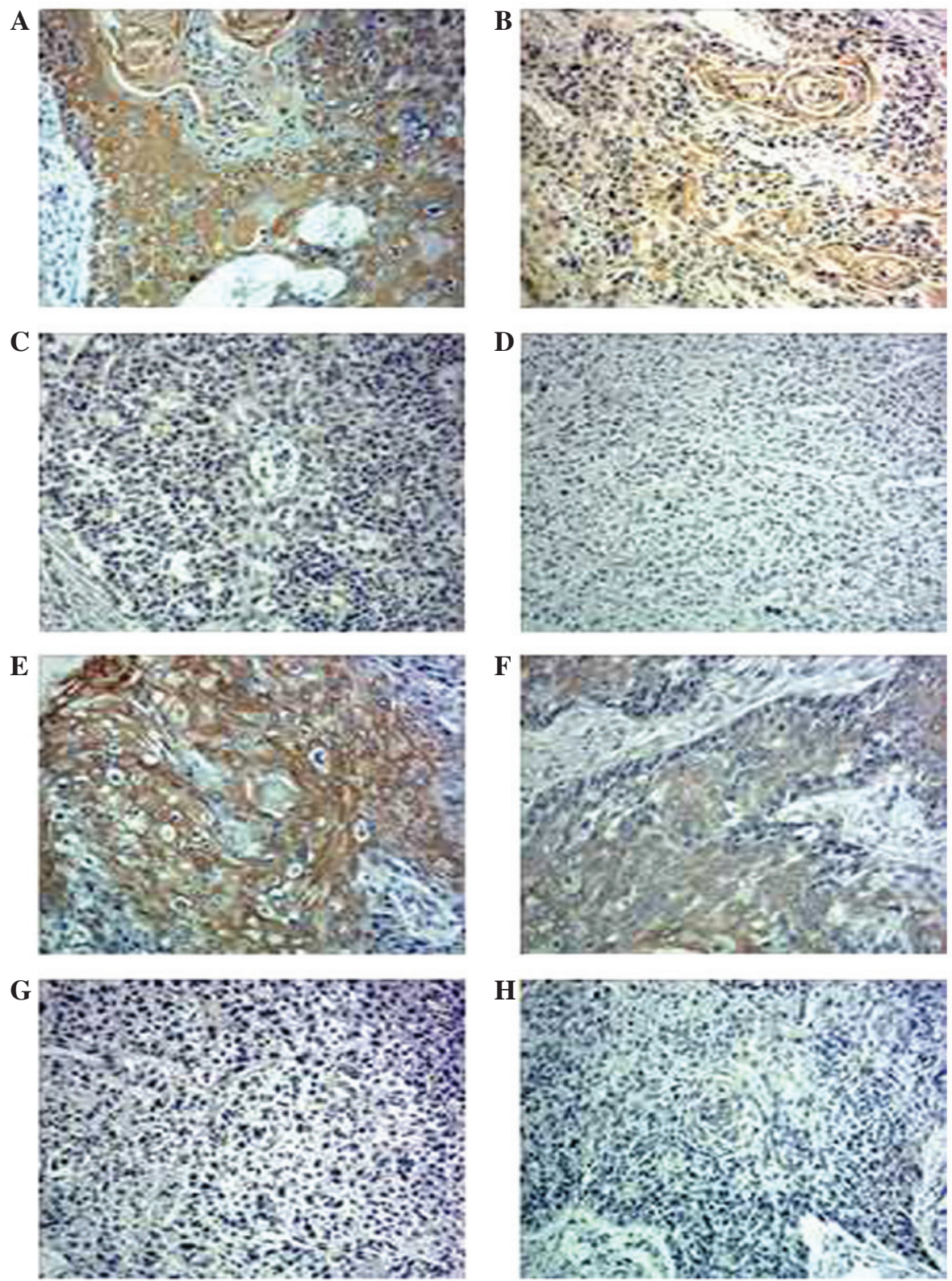

Figure 1. Immunohistochemical staining of human telomerase reverse transcriptase (hTERT) and ubiquitin-conjugating enzyme E2D 3 (UBE2D3) protein in esophageal cancer tissues. Representative examples of (A-B) high and (C-D) low hTERT expression, and (E-F) high and (G-H) low UBE2D3 expression (magnification, x100).

UBE2D3 expression was $\mathrm{r}=-0.18(\mathrm{P}=0.027)$, indicating that hTERT and UBE2D3 expression are negatively correlated with each other.

\section{Discussion}

The present study investigated 150 esophageal cancer and 30 adjacent tissues to evaluate the prognostic value of a number of conventional clinicopathological and cellular molecular factors. In accordance with previously conducted studies (24-26), univariate analysis demonstrated that traditional clinical parameters, such as T, N and overall TNM stage, were important factors for predicting the survival rate, whereas tumor histology and adjuvant therapy were not (Table II). However, whether tumor location affects the prognosis of esophageal cancer is a controversial topic. Yang et al (27) demonstrated that the tumor location did not impact the survival rate of esophageal cancer; however, in the seventh edition of the UICC TNM system (20), tumor location (upper and middle thoracic versus lower thoracic) was important for grouping T2-3NOM0 squamous cell cancers. The present univariate and multivariate analysis indicated that tumor location (upper versus middle and lower thoracic) was associated with survival rate and may be an independent prognostic factor in esophageal cancer. In addition, the present study identified that lymph node involvement may be an independent prognostic factor for esophageal cancer, which was consistent with the results of previous studies $(28,29)$.

hTERT confers limitless replicative potential to cancer cells (30), and previous studies have established immortalized human esophageal epithelial cell models by the introduction of hTERT (31). Furthermore, hTERT is able to promote the development of invasive esophageal squamous cell cancer by 
Table II. Association between hTERT and UBE2D3 expression levels, and various clinicopathological characteristics.

\begin{tabular}{|c|c|c|c|c|c|c|}
\hline \multirow[b]{2}{*}{ Characteristic } & \multicolumn{3}{|c|}{ hTERT expression } & \multicolumn{3}{|c|}{ UBE2D3 expression } \\
\hline & $\begin{array}{l}\text { Low, n (\%) } \\
\quad(\mathrm{n}=59)\end{array}$ & $\begin{array}{l}\text { High, n (\%) } \\
\quad(\mathrm{n}=91)\end{array}$ & P-value & $\begin{array}{c}\text { Low, n (\%) } \\
\quad(\mathrm{n}=95)\end{array}$ & $\begin{array}{l}\text { High, } \mathrm{n}(\%) \\
\quad(\mathrm{n}=55)\end{array}$ & P-value \\
\hline Age, years & & & 0.067 & & & 0.651 \\
\hline$<60$ & $33(47.1)$ & $37(52.9)$ & & $43(61.4)$ & $27(38.6)$ & \\
\hline$\geq 60$ & $26(32.5)$ & $54(67.5)$ & & $52(65.0)$ & $28(35.0)$ & \\
\hline Gender & & & 0.154 & & & 0.811 \\
\hline Male & $52(41.9)$ & $72(58.1)$ & & $78(62.9)$ & $46(37.1)$ & \\
\hline Female & $7(26.9)$ & $19(73.1)$ & & $17(65.4)$ & $9(34.6)$ & \\
\hline Location & & & 0.900 & & & 0.261 \\
\hline Upper thoracic & $8(38.1)$ & $13(61.9)$ & & $11(52.4)$ & $10(47.6)$ & \\
\hline Middle/lower thoracic & $51(39.5)$ & $78(60.5)$ & & $84(65.1)$ & $45(34.9)$ & \\
\hline Tumor size, $\mathrm{cm}$ & & & 0.039 & & & 0.262 \\
\hline$<5$ & $38(46.9)$ & $43(53.1)$ & & $48(59.3)$ & $33(40.7)$ & \\
\hline$\geq 5$ & $21(30.4)$ & $48(69.6)$ & & $47(68.1)$ & $22(31.9)$ & \\
\hline Histological grade ${ }^{a}$ & & & 0.032 & & & 0.007 \\
\hline $\mathrm{G} 1 / 2$ & $52(43.7)$ & $67(56.3)$ & & $64(57.1)$ & $48(42.9)$ & \\
\hline $\mathrm{G} 3 / 4$ & 7 (22.6) & $24(77.4)$ & & $31(81.6)$ & $7(18.4)$ & \\
\hline T stage & & & 0.030 & & & 0.154 \\
\hline $\mathrm{T} 1-2$ & $32(49.2)$ & $33(50.8)$ & & $37(56.9)$ & $28(43.1)$ & \\
\hline T3-4 & $27(45.8)$ & $58(63.7)$ & & $58(68.2)$ & $27(31.8)$ & \\
\hline $\mathrm{N}$ stage & & & 0.018 & & & 0.016 \\
\hline No & $37(48.7)$ & $39(51.3)$ & & $41(53.9)$ & $35(46.1)$ & \\
\hline N1-3 & $22(29.7)$ & $52(70.3)$ & & $54(73.0)$ & $20(27.0)$ & \\
\hline TNM stage & & & 0.012 & & & 0.347 \\
\hline Early (I-II) & $42(47.7)$ & $46(52.3)$ & & $53(60.2)$ & $35(39.8)$ & \\
\hline Advanced (III-IV) & $17(27.4)$ & $45(72.6)$ & & $42(67.7)$ & $20(32.3)$ & \\
\hline Recurrence & & & 0.09 & & & 0.008 \\
\hline No & $31(47.0)$ & $35(53.0)$ & & $34(51.5)$ & $32(48.5)$ & \\
\hline Yes & $28(33.3)$ & $56(66.7)$ & & $61(72.6)$ & $23(27.4)$ & \\
\hline Recurrence location ${ }^{\mathrm{b}}$ & & & 0.677 & & & 0.731 \\
\hline Local-regional & $16(32.0)$ & $34(68.0)$ & & $37(74.0)$ & $13(26.0)$ & \\
\hline Distant & $12(35.3)$ & $22(64.7)$ & & $24(70.6)$ & $10(29.4)$ & \\
\hline
\end{tabular}

${ }^{\mathrm{a}} \mathrm{G} 1 / 2$ were defined as well- or moderately-differentiated and G3/4 were defined as poorly-differentiated or undifferentiated. ${ }^{\mathrm{b}}$ Analysis was based on 84 recurrent cases of esophageal cancer. hTERT, human telomerase reverse transcriptase; UBE2D3, ubiquitin-conjugating enzyme E2D 3; TNM, tumor-node-metastasis.

interacting with epidermal growth factor receptor and p53 (32). Telomerase activity has been extensively studied in various types of malignant tumor for clinical, diagnostic and/or prognostic purposes $(12,13,33)$, and it has been proposed for use as a marker of poor prognosis in such tumors. The present study determined that hTERT was more frequently elevated in the esophageal cancer tissues compared with the adjacent healthy tissues. In the cancer tissues, the expression of hTERT was also elevated in tumors with large size, poor differentiation, deep tumor invasion, lymph node metastasis and advanced TNM stage. Furthermore, strong expression of hTERT was correlated with OS time, indicating that hTERT participates in the progress of esophageal cancer and may be a poor prognostic biomarker of esophageal cancer tumors. However, in multivariate analysis, hTERT expression was not an independent prognostic factor, therefore, a combination test of telomerase activity with other prognostic factors may be necessary.

UBE2D3 is a member of E2 family and is a crucial component of the ubiquitination cascade, acting as a key mediator of the interaction between E1 and E3 $(34,35)$. The whole ubiquitination process is responsible for $80 \%$ of proteasomal cellular protein degradation. Upregulation of UBE2D3 in acute promyelocytic leukemia cells leads to the ubiquitination of cyclin D1 and its degradation in the proteasome (36). However, in the absence of UBE2D3, cyclin D1 is not degraded and tumor cells continue to cycle (37). Mittal et al (38) reported that knocking down 
Table III. Univariate analysis of factors regarding overall survival.

\begin{tabular}{|c|c|c|c|c|}
\hline Characteristic & $\mathrm{n}(\%)$ & Five-year survival rate, $\%$ & $\chi^{2 \mathrm{a}}$ & P-value \\
\hline Age, years & & & 0.459 & 0.498 \\
\hline$<60$ & $70(46.7)$ & 29.0 & & \\
\hline$\geq 60$ & $80(53.3)$ & 22.0 & & \\
\hline Gender & & & 0.068 & 0.405 \\
\hline Male & $124(82.7)$ & 28.0 & & \\
\hline Female & $26(17.3)$ & 17.0 & & \\
\hline Location & & & 4.455 & 0.035 \\
\hline Upper thoracic & $21(14.0)$ & 11.0 & & \\
\hline Middle/lower thoracic & $129(86.0)$ & 30.0 & & \\
\hline Tumor size, $\mathrm{cm}$ & & & 3.812 & 0.051 \\
\hline$<5$ & $81(54.0)$ & 29.8 & & \\
\hline$\geq 5$ & $69(46.0)$ & 20.2 & & \\
\hline Histological grade ${ }^{b}$ & & & 0.068 & 0.795 \\
\hline $\mathrm{G} 1 / 2$ & $119(79.3)$ & 27.0 & & \\
\hline $\mathrm{G} 3 / 4$ & $31(20.7)$ & 19.0 & & \\
\hline T stage & & & 4.278 & 0.039 \\
\hline $\mathrm{T} 1-\mathrm{T} 2$ & $65(43.3)$ & 35.5 & & \\
\hline T3-T4 & $85(56.7)$ & 15.0 & & \\
\hline $\mathrm{N}$ stage & & & 11.290 & 0.001 \\
\hline N0 & $76(50.7)$ & 35.8 & & \\
\hline $\mathrm{N} 1-3$ & $74(49.3)$ & 9.1 & & \\
\hline TNM stage & & & 16.000 & $<0.001$ \\
\hline Early (I-II) & $88(58.7)$ & 34.9 & & \\
\hline Advanced (III-IV) & $62(41.3)$ & 17.0 & & \\
\hline Adjuvant radiotherapy & & & 0.293 & 0.588 \\
\hline Yes & $40(26.7)$ & 26.0 & & \\
\hline No & $110(73.3)$ & 23.0 & & \\
\hline Adjuvant chemotherapy & & & 0.004 & 0.951 \\
\hline Yes & $70(46.7)$ & 26.1 & & \\
\hline No & $80(53.3)$ & 24.8 & & \\
\hline hTERT expression & & & 6.353 & 0.012 \\
\hline Low & $59(39.3)$ & 35.0 & & \\
\hline High & $91(60.7)$ & 19.5 & & \\
\hline UBE2D3 expression & & & 9.145 & 0.002 \\
\hline Low & $95(63.3)$ & 15.7 & & \\
\hline High & $55(36.7)$ & 40.9 & & \\
\hline
\end{tabular}

${ }^{a}$ Determined by performing a log-rank test. ${ }^{b} \mathrm{G} 1 / 2$ were defined as well- or moderately-differentiated and G3/4 were defined as poorly-differentiated or undifferentiated. TNM, tumor-node-metastasis; hTERT, human telomerase reverse transcriptase; UBE2D3, ubiquitin-conjugating enzyme E2D 3.

UBE2D3 in human breast cancer cells resulted in elevated cyclin D1 levels, and that a low level of UBE2D3 expression was a determinant factor in the progression of metastatic breast cancer. These two studies indicated that UBE2D3 expression is involved in cell cycle regulation via the degradation of cyclin D1; in consideration of this biological behavior, the present study proposes that UBE2D2 expression levels may promote tumor development. Furthermore, the current study identified that the expression of UBE2D3 was significantly lower in the esophageal cancer tissues compared with the adjacent healthy tissues, as well as significantly lower in the cancer tissues with lymph node involvement and poor differentiation. In addition, UBE2D3 appeared to be an independent prognostic factor for esophageal cancer. Thus, it is proposed that UBE2D3 expression may be involved in the progression of esophageal cancer.

Most notably, Spearman correlation coefficient analysis revealed a negative correlation between UBE2D3 and hTERT protein expression levels, which was consistent with 
Table IV. Multivariate Cox proportional hazards analysis of overall survival.

\begin{tabular}{lccr}
\hline & \multicolumn{3}{c}{ Overall survival } \\
\cline { 2 - 4 } Factor $^{\mathrm{n}}$ & $\mathrm{HR}$ & $95 \%$ CI & P-value \\
\hline Tumor location & 0.476 & $0.270-0.841$ & 0.011 \\
T stage & 1.086 & $0.674-1.754$ & 0.734 \\
N stage & 1.694 & $1.055-2.721$ & 0.029 \\
hTERT expression & 1.589 & $0.990-2.551$ & 0.055 \\
UBE2D3 expression & 0.487 & $0.293-0.807$ & 0.005 \\
\hline
\end{tabular}

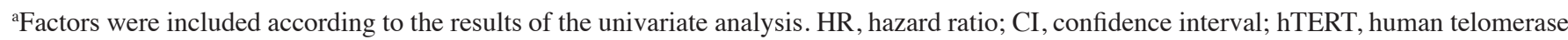
reverse transcriptase; UBE2D3, ubiquitin-conjugating enzyme E2D 3.
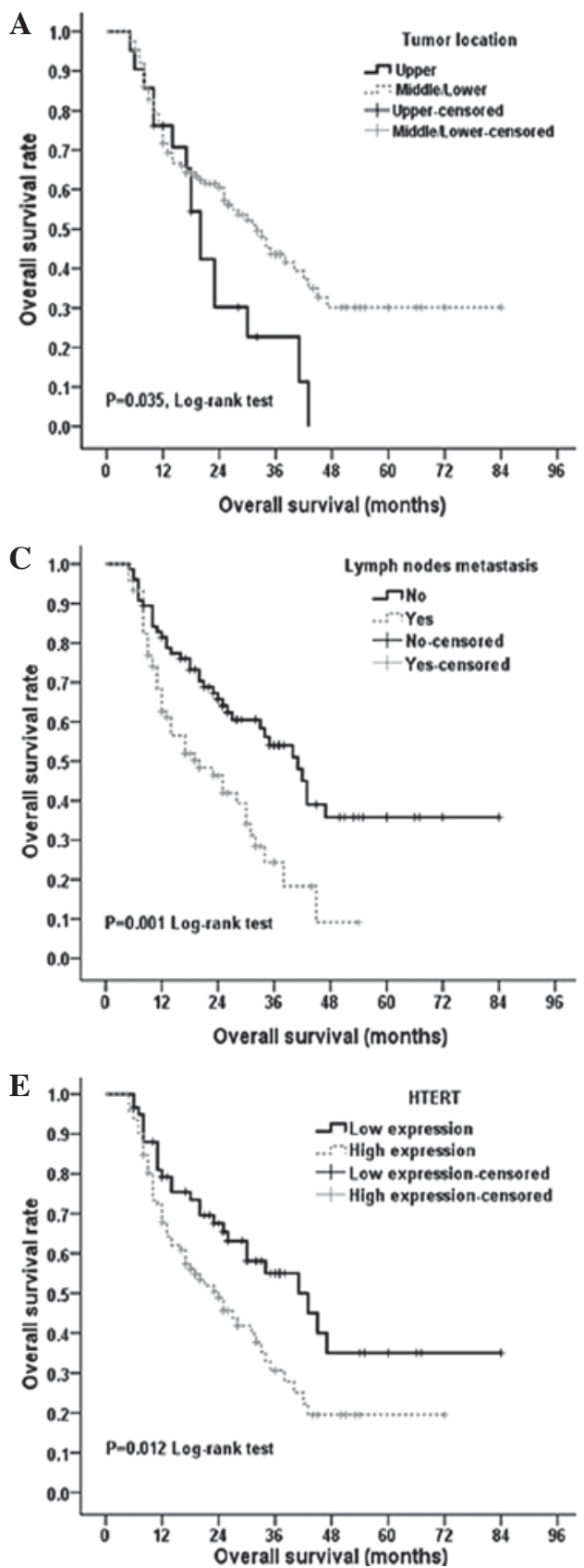
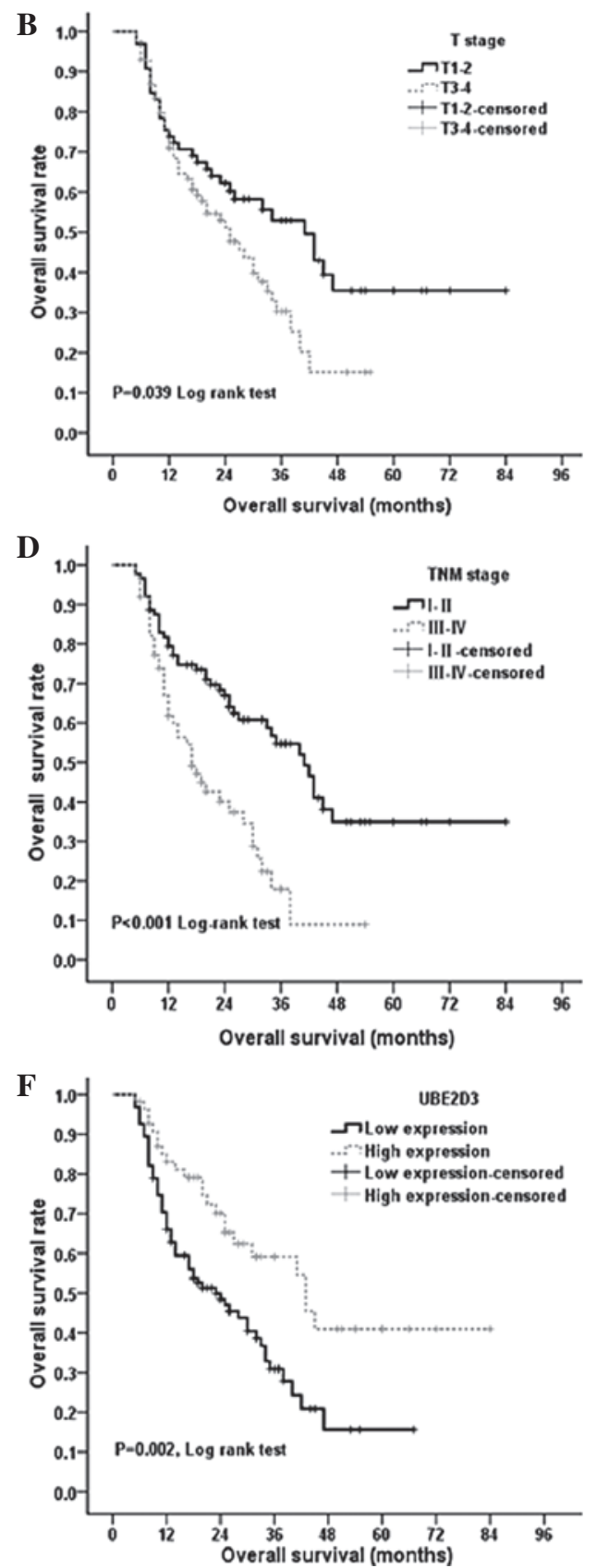

Figure 2. Cumulative overall survival (OS) of esophageal cancer patients. (A) A higher tumor location was associated with poorer OS. (B-D) Advanced T stage, lymph node status and TNM stage were associated with poor OS. (E) Patients in the high hTERT expression group were at a higher risk of mortality. (F) Patients in the UBE2D3 low expression group were at a higher risk of mortality. TNM, tumor-node-metastasis; hTERT, human telomerase reverse transcriptase; UBE2D3, ubiquitin-conjugating enzyme E2D 3. 
a previous study (19). These results indicate that hTERT and UBE2D3 may interact with each other, validating the proposal that UBE2D3 potentially has a role in the hTERT signaling pathway. However, the mechanism by which the ubiquitination process of UBE2D3 is involved in the interaction with the hTERT pathway, and whether UBE2D3 expression exists as a universal phenomenon in all types of tumor, requires additional studies to be conducted in the future.

In conclusion, the present study demonstrated that hTERT and UBE2D3 expression are negatively correlated, and that the two proteins demonstrate a strong association with the prognosis in esophageal cancer. Furthermore, the expression of UBE2D3, lymph node involvement and tumor location were independent predictive prognostic factors; thus, UBE2D3 expression may be a promising prognostic biomarker in esophageal cancer. However, the current study was based on retrospective analysis and semi-quantitative research, therefore, prospective randomized clinical trials and basic quantitative research are required to evaluate the clinical utility of the present study results.

\section{Acknowledgements}

The present study was sponsored by the National Natural Science foundation of china (grant no. 81071825) and the Fundamental Research Funds for the Central Universities (grant no. 2042014kf0114).

\section{References}

1. Yang $\mathrm{H}$ and Chen YX: Improvement analysis of article quality in World Journal of Gastroenterology during 2008-2012. World J Gastroenterol 19: 7830-7835, 2013.

2. Mao WM, Zheng WH and Ling ZQ: Epidemiologic risk factors for esophageal cancer development. Asian Pac J Cancer Prev 12: 2461-2466, 2011.

3. Wang X, Fan JC, Wang AR, et al: Epidemiology of esophageal cancer in Yanting - regional report of a national screening programme in China. Asian Pac J Cancer Prev 14: 2429-2432, 2013.

4. Lin Y, Totsuka Y, He Y, et al: Epidemiology of esophageal cancer in Japan and China. J Epidemiol 23: 233-242, 2013.

5. Zhang Y: Epidemiology of esophageal cancer. World J Gastroenterol 19: 5598-5606, 2013.

6. Gertler R, Stein HJ, Langer R, et al: Long-term outcome of 2920 patients with cancers of the esophagus and esophagogastric junction: evaluation of the New Union Internationale Contre le Cancer/American Joint Cancer Committee staging system. Ann Surg 253: 689-698, 2011.

7. Lu W, Zhang Y, Liu D, et al: Telomeres - structure, function, and regulation. Exp Cell Res 319: 133-141, 2013.

8. Philippi C, Loretz B, Schaefer UF and Lehr CM: Telomerase as an emerging target to fight cancer - opportunities and challenges for nanomedicine. J Control Release 146: 228-240, 2010.

9. Counter CM, Meyerson M, Eaton EN, et al: Telomerase activity is restored in human cells by ectopic expression of hTERT (hEST2), the catalytic subunit of telomerase. Oncogene 16: 1217-1222, 1998.

10. Ulaner GA, Hu JF, Vu TH, et al: Telomerase activity in human development is regulated by human telomerase reverse transcriptase (hTERT) transcription and by alternate splicing of hTERT transcripts. Cancer Res 58: 4168-4172, 1998.

11. Proctor A, Brownhill SC and Burchill SA: The promise of telomere length, telomerase activity and its regulation in the translocation-dependent cancer ESFT; clinical challenges and utility. Biochim Biophys Acta 1792: 260-274, 2009.

12. Simsek BC, Pehlivan S and Karaoglu A: Human telomerase reverse transcriptase expression in colorectal tumors: correlations with immunohistochemical expression and clinicopathologic features. Ann Diagn Pathol 14: 413-417, 2010.

13. Wang G, Wang W, Zhou J and Yang X: Correlation between telomerase activity and matrix metalloproteinases 2 expression in gastric cancer. Cancer Biomark 13: 21-28, 2013.
14. Poremba C, Heine B, Diallo R, et al: Telomerase as a prognostic marker in breast cancer: high-throughput tissue microarray analysis of hTERT and hTR. J Pathol 198: 181-189, 2002.

15. Tu Y, Chen C, Pan J, et al: The Ubiquitin Proteasome Pathway (UPP) in the regulation of cell cycle control and DNA damage repair and its implication in tumorigenesis. Int J Clin Exp Pathol 5: 726-738, 2012.

16. Amm I, Sommer T and Wolf DH: Protein quality control and elimination of protein waste: the role of the ubiquitin-proteasome system. Biochim Biophys Acta 1843: 182-196, 2014.

17. Clague MJ and Urbé S: Ubiquitin: same molecule, different degradation pathways. Cell 143: 682-685, 2010.

18. Kleiger G and Mayor T: Perilous journey: a tour of the ubiquitin-proteasome system. Trends Cell Biol 24: 352-359, 2014.

19. Wang W, Yang L, Hu L, et al: Inhibition of UBE2D3 expression attenuates radiosensitivity of MCF-7 human breast cancer cells by increasing hTERT expression and activity. PLoS One 8: e64660, 2013.

20. Sobin LH, Gospodarowicz MK and Wittekind C (eds): TNM Classification of Malignant Tumours. 7th edition. Wiley-Blackwell, West Sussex, 2009.

21. Yang $\mathrm{CH}$, Hung WC, Wang SL, et al: Immunoexpression and prognostic role of hTERT and cyclin D1 in urothelial carcinoma. APMIS 116: 309-316, 2008.

22. Fujita T,Ikeda H, Kawasaki K, et al: Clinicopathological relevance of UbcH10 in breast cancer. Cancer Sci 100: 238-248, 2009.

23. Kumaki F, Kawai T, Hiroi S, et al: Telomerase activity and expression of human telomerase RNA component and human telomerase reverse transcriptase in lung carcinomas. Hum Pathol 32: 188-195, 2001

24. Yam PC, Tong D and Law S: Comparisons of sixth and seventh edition of the American Joint Cancer Committee staging systems for esophageal cancer. Ann Surg Oncol 21: 583-588, 2014.

25. Hsu PK, Wu YC, Chou TY, et al: Comparison of the 6th and 7th editions of the American Joint Committee on Cancer tumor-node-metastasis staging system in patients with resected esophageal carcinoma. Ann Thorac Surg 89: 1024-1031, 2010.

26. Hong JC, Murphy JD, Wang SJ, et al: Chemoradiotherapy before and after surgery for locally advanced esophageal cancer: a SEER-Medicare analysis. Ann Surg Oncol 20: 3999-4007, 2013.

27. Yang HX, Hou X, Liu QW, et al: Tumor location does not impact long-term survival in patients with operable thoracic esophageal squamous cell carcinoma in China. Ann Thorac Surg 93: 1861-1866, 2012.

28. Marjanovic G, Schricker M, Walch A, et al: Detection of lymph node involvement by cytokeratin immunohistochemistry is an independent prognostic factor after curative resection of esophageal cancer. J Gastrointest Surg 15: 29-37, 2011.

29. Dhupar R, Correa AM, Ajani J, et al: Concordance of studies for nodal staging is prognostic for worse survival in esophageal cancer. Dis Esophagus 27: 770-776, 2013.

30. Vallböhmer D, Brabender J, Metzger R and Hölscher AH: Genetics in the pathogenesis of esophageal cancer: possible predictive and prognostic factors. J Gastrointest Surg 14 (Suppl 1): S75-S80, 2010.

31. Cheung PY, Deng W, Man C, et al: Genetic alterations in a telomerase-immortalized human esophageal epithelial cell line: Implications for carcinogenesis. Cancer Lett 293: 41-51, 2010.

32. Okawa T, Michaylira CZ, Kalabis J, et al: The functional interplay between EGFR overexpression, hTERT activation, and p53 mutation in esophageal epithelial cells with activation of stromal fibroblasts induces tumor development, invasion, and differentiation. Genes Dev 21: 2788-2803, 2007.

33. Sakabe R, Murakami Y, Uemura K, et al: Prognostic significance of telomerase activity and human telomerase reverse transcriptase expression in ampullary carcinoma. Ann Surg Oncol 19: 3072-3080, 2012.

34. Page RC, Pruneda JN, Amick J, et al: Structural insights into the conformation and oligomerization of E2 ubiquitin conjugates. Biochemistry 51: 4175-4187, 2012.

35. Ye Y and Rape M: Building ubiquitin chains: E2 enzymes at work. Nat Rev Mol Cell Biol 10: 755-764, 2009.

36. Adams J: The proteasome: structure, function, and role in the cell. Cancer Treat Rev 29 (Suppl 1): 3-9, 2003.

37. Khanna-Gupta A and Berliner N: ATRA: Finding targeted APL therapy targets. Blood 110: 476-477, 2007.

38. Mittal MK, Singh K, Misra S and Chaudhuri G: SLUG-induced elevation of D1 cyclin in breast cancer cells through the inhibition of its ubiquitination. J Biol Chem 286: 469-479, 2011. 